University of Nebraska - Lincoln

DigitalCommons@University of Nebraska - Lincoln

Faculty Publications from the Department of Electrical \& Computer Engineering, Department Electrical and Computer Engineering

2001

\title{
Polarimetric Processing of Coherent Random Noise Radar Data for Buried Object Detection
}

\author{
Yi Xu \\ University of Nebraska-Lincoln \\ Ram M. Narayanan \\ University of Nebraska-Lincoln \\ Xiojian Xu \\ University of Nebraska-Lincoln \\ John O. Curtis \\ University of Nebraska-Lincoln
}

Follow this and additional works at: https://digitalcommons.unl.edu/electricalengineeringfacpub

Part of the Electrical and Computer Engineering Commons

Xu, Yi; Narayanan, Ram M.; Xu, Xiojian; and Curtis, John O., "Polarimetric Processing of Coherent Random Noise Radar Data for Buried Object Detection" (2001). Faculty Publications from the Department of Electrical and Computer Engineering. 148.

https://digitalcommons.unl.edu/electricalengineeringfacpub/148

This Article is brought to you for free and open access by the Electrical \& Computer Engineering, Department of at DigitalCommons@University of Nebraska - Lincoln. It has been accepted for inclusion in Faculty Publications from the Department of Electrical and Computer Engineering by an authorized administrator of DigitalCommons@University of Nebraska - Lincoln. 


\title{
Polarimetric Processing of Coherent Random Noise Radar Data for Buried Object Detection
}

\author{
Yi Xu, Ram M. Narayanan, Fellow, IEEE, Xiaojian Xu, and John O. Curtis
}

\begin{abstract}
Random noise polarimetry is a new radar technique for high-resolution probing of subsurface objects and interfaces. The University of Nebraska has developed a polarimetric random noise radar system based on the heterodyne correlation technique. Simulation studies and performance tests on the system confirm its ability to respond to phase differences in the received signals. In addition to polarimetric processing capability and the simplified system design, random noise radar also possesses other desirable features, such as immunity from radio frequency interference (RFI). The paper discusses the theoretical foundations of random noise polarimetry, and presents examples out of the entire data set collected that demonstrate the usefulness of the image processing and Stokes matrix presentation to enhance target detection using the coherent random noise radar.
\end{abstract}

Index Terms-Polarimetry, random noise radar, subsurface radar, ultrawideband.

\section{INTRODUCTION}

$\mathbf{T}$ HE USE of radar techniques to detect, locate, and identify buried shallow subsurface objects is of considerable interest in recent years. Various kinds of radars, including impulse, chirp-pulse, coded-pulse, FM-CW, and step-frequency CW operation systems, have been developed [1]-[5]. For such applications, the use of wideband random noise transmit waveforms afford simplified system designs while yielding good depth resolution [6] and also being exempt from radio frequency interference (RFI) at lower radar bands.

The University of Nebraska, Lincoln, has developed a polarimetric random noise radar system used mainly for detecting shallowly buried minelike objects. Simulation studies and performance tests on the system confirm its ability to respond to phase differences in the received signal despite the fact of the probing waveform in random noise. This ground penetrating radar (GPR) system uses a wide bandwidth random noise signal operating within the $1-2 \mathrm{GHz}$ frequency range. High spatial resolution in the depth (range) dimension is achieved due to the wide bandwidth of the transmit signal. The polarimetric random noise radar system was used to gather data from a variety of buried targets at different depths and with different relative orientations. A detailed description of the noise radar system and some simulation and preliminary experimental results have been

Manuscript received November 9, 1999; revised July 10, 2000. This work was supported by Contract DACA39-93-K-0031 awarded by the U.S. Army Waterways Experiment Station.

Y. Xu, R. M. Narayanan, and X. Xu are with the Department of Electrical Engineering, Center for Electro-Optics, University of Nebraska, Lincoln, NE 68588-0511 USA.

J. O. Curtis is with the Environmental Laboratory, U.S. Army Waterways Experiment Station, Vicksburg, MS 39180-6199 USA.

Publisher Item Identifier S 0196-2892(01)02089-7. published in [7]. In this paper, we present examples from the entire data set collected that demonstrate the usefulness of the image processing and Stokes matrix presentation to enhance target detection for the coherent random noise GPR.

The remainder of this paper is organized as follows. Section II gives a brief description of the coherent ultra-wideband random noise radar system. A simplified theoretical development of the random noise polarimetry is found in Section III. In Section IV, we present the Stokes matrix image processing results as well as several image preprocessing techniques such as apodization filtering, smoothing, and thresholding algorithms, which are implemented in order to suppress the range sidelobes and enhance detection. We conclude the paper in Section V.

\section{DESCRIPTION OF COHERENT UlTRAWIDEBAND RANDOM NOISE RADAR SYSTEM}

A block diagram of the system is shown in Fig. 1. Relevant system specifications are listed in Table I. The noise source produces a signal having a Gaussian amplitude distribution and a constant power spectral density in the $1-2 \mathrm{GHz}$ frequency range. This signal is divided into two in-phase components by a $3-\mathrm{dB}$ in-phase power divider. One component is fed to an amplifier which has a power output of greater than $+40 \mathrm{dBm}$ at its $1-\mathrm{dB}$ gain compression point. The average power output of the amplifier is $+30 \mathrm{dBm}(1 \mathrm{~W})$, and it can thus amplify noise spikes that are as high as $10 \mathrm{~dB}$ above the mean noise power. The output of this amplifier is connected to either the $\mathrm{V}$ or $\mathrm{H}$ polarization input on the dual-polarized broadband log-periodic transmit antenna.

The second component is connected to a combination of a fixed and a digitally controlled variable delay line. The fixed delay line serves to ensure that the correlation operation is performed in a manner as to avoid antenna-to-antenna direct coupling. The variable delay provides the delay of the transmit signal in order to correlate it with the received signal from objects or interfaces at that depth corresponding to that delay. The delay line can be programmed for delays from 0 to $19.84 \mathrm{~ns}$ in $0.156 \mathrm{~ns}$ steps. This step size ensures that the depth resolution of the system as governed by its $1-\mathrm{GHz}$ bandwidth is not compromised. The output of the delay line is then mixed with a $160-\mathrm{MHz}$ coherent stable signal from a phase lock oscillator. This is done in order to perform coherent processing of the received noise signals. The output from this mixer is divided, amplified, and then fed into the co-polarized and cross-polarized receive channels. The co-polarized channel corresponds to the channel connected to the same antenna polarization as the transmit antenna. The cross-polarized channel is orthogonal to 


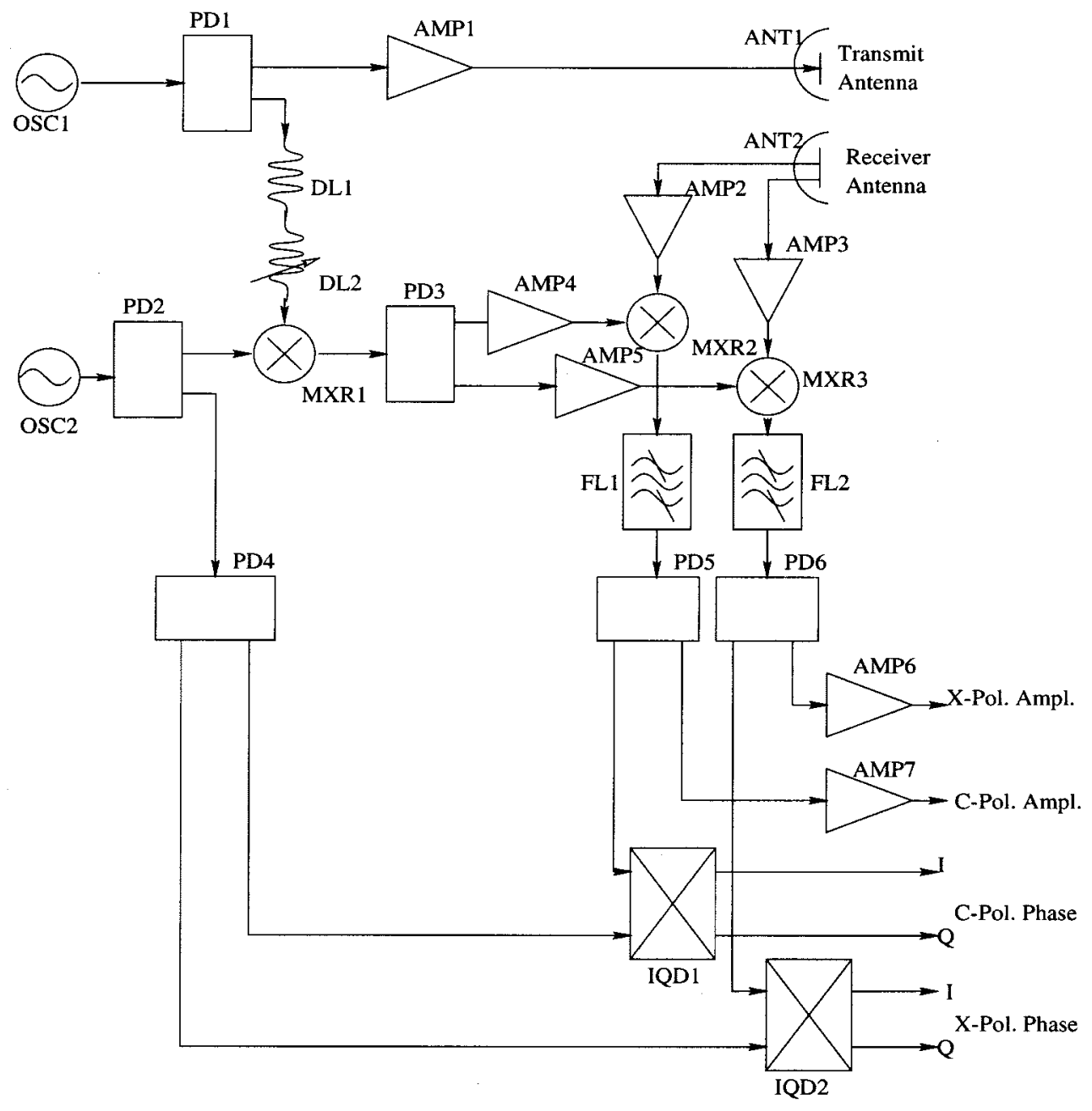

Fig. 1. Block diagram of the coherent polarimetric ultrawideband random noise radar system.

TABLE I

POLARIMETRIC RANDOM NOISE RADAR SYSTEM SPECIFICATIONS

\begin{tabular}{|c|c|}
\hline Paramiler & Spetificatisa \\
\hline Fonquacy Rarge & 1-2 GHz \\
\hline Syatem IF & $160 \mathrm{MHz}$ \\
\hline Syotem Tranenit Wavoform & Costinucen Wave \\
\hline Modelation & Gsossian Naise \\
\hline Trassmit Power & 1 Watt $(30 \mathrm{dBa})$ Average \\
\hline Receiver Detoctalle Poser & $-92 \mathrm{dBn}$ Minimin= \\
\hline Aateraa & Broedland Duel Polarized Log Periodic \\
\hline Antenna 3-dB Nominal Beammidth & 60" E-Plase and $100^{\circ}$ H-Plaze \\
\hline Trasumitter Polarization & Vertiral or Beriocntal (Munual Switchable) \\
\hline Rrosiver Polarization & Bceh Vertical and Horizcatal \\
\hline Syatem Delay Tims & $0-19.84$ nexc in 128 stepes of $0.156 \mathrm{macc}$ \\
\hline Syctem Resolution & 3-8.6 cu (Depends on Soll Moisture) \\
\hline
\end{tabular}

the transmit antenna polarization. The noise signal in each receiver chain is amplified and then mixed with the coherent noise signal. The output of the mixer is passed through a bandpass filter and then divided by a $3-\mathrm{dB}$ power divider. One output goes to a logarithmic amplifier, which ensures that a wide range of scattered power levels can be processed. The second output goes to the inputs of the I/Q detector, from where the I and Q outputs can be related to the polarimetric co-polarized or cross-polarized scattering characteristics of the buried object or interface.

The current coherent random radar system provides a simplified system design while preserving all the advantages and avoiding the drawbacks that impulse and linear frequency-modulated (LFM) or step-frequency CW GPR systems possess. These include yielding high depth resolution, preservation of scattering phase characteristics, and immunity from the impacts of antenna coupling and RFI, and so on. For example, since the usual ultra-wideband GPR has much broader bandwidth than conventional radar and operates at lower radar frequency bands, it is extremely vulnerable to RFI. The GPR with its higher resolution requirement must reject these RFI signals by using a notch filter or other adaptive filtering techniques. This causes an obvious increase of the range sidelobes. On the contrary, the random noise radar is immune from the impact of such RFI signals. This is due to the fact that since the RFI signals are uncorrelated with the transmit waveform, any mixing operation at the receiver of the noise radar system will ideally produce an output voltage of zero. Fig. 2 compares the performance of a LFM and a random noise radar operating over the same bandwidth under RFI conditions using simulation. This simulation shows that, when twenty percent of the radar bandwidth is interfered by RFI, with an integration number 

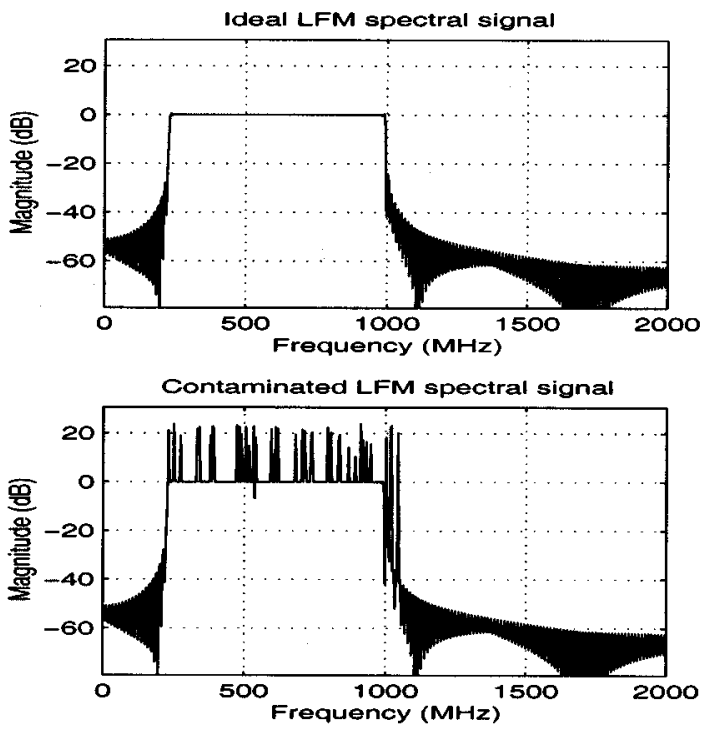

(a)
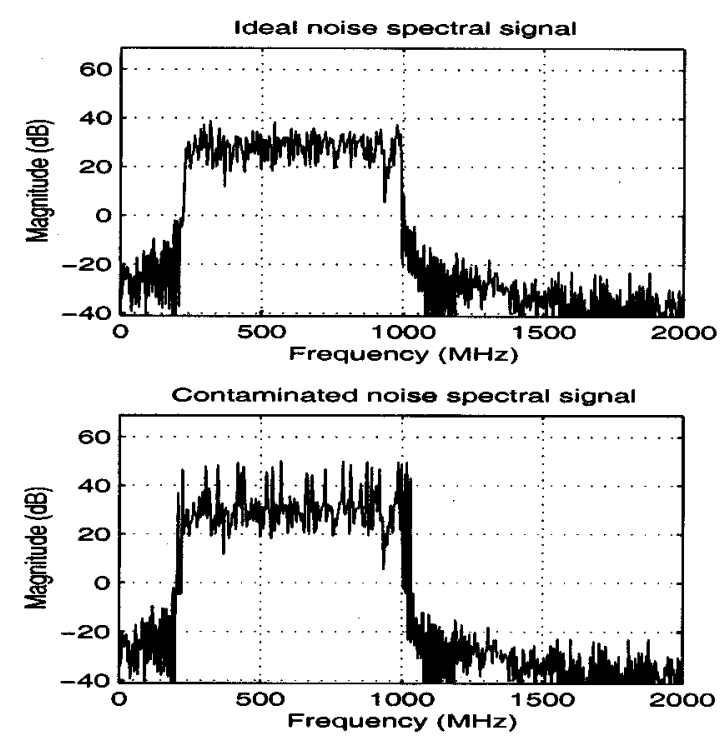

(b)

Fig. 2. Impact of RFI on (a) UWB LFM radar and (b) UWB random noise radar.

of 512, a random noise radar will have at least a 10-dB lower sidelobe level than a conventional LFM radar.

\section{THEORY OF RANDOM NOISE POLARIMETRY}

Assuming that the transmitted signal has a Gaussian amplitude distribution and a uniform power spectral density, we model the transmit voltage wave $v_{t}(t)$ as

$$
v_{t}(t)=a(t) \cos \left(\omega_{0}+\delta \omega\right) t
$$

where $a(t)$ takes into account the amplitude distribution, and $\delta \omega(t)$ takes into account the frequency spectrum of $v_{t}(t) . \omega_{0}$ is the center frequency of transmission. We assume that $a(t)$ and $\delta \omega(t)$ are ergodic processes. Furthermore, we assume that $a(t)$ and $\delta \omega(t)$ are uncorrelated and statistically independent.
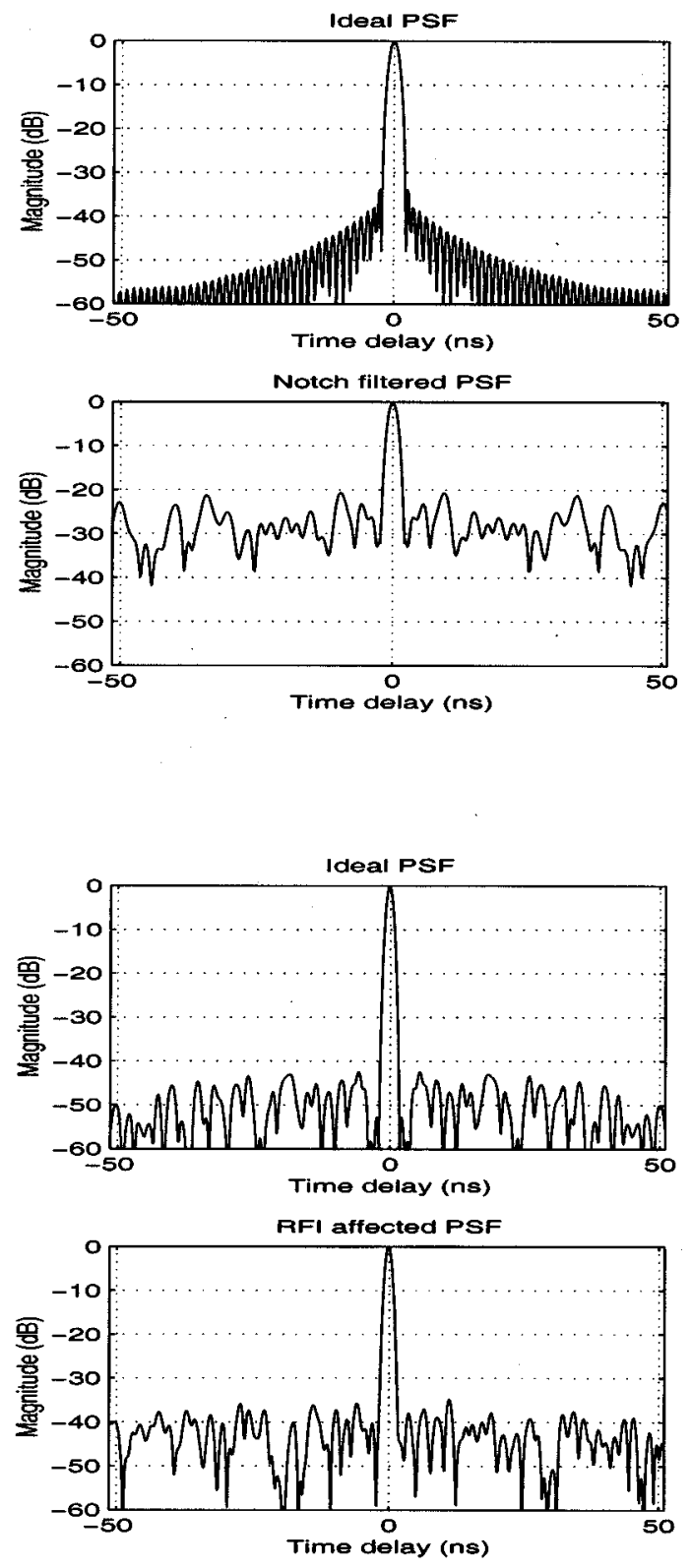

If $R_{0}$ is the characteristic impedance of the system, the average power transmitted $P_{t}$ is given by

$$
P_{t}=\frac{\overline{v_{t}^{2}(t)}}{R_{0}}
$$

which can be shown to reduce to [7]

$$
P_{t}=\frac{1}{2 R_{0}} \overline{a^{2}(t)} .
$$

Let $\epsilon_{r}\left(=\epsilon_{r}^{\prime}-j \epsilon_{r}^{\prime \prime}\right)$ be the complex dielectric constant of the soil medium, from which the attenuation constant $\alpha$, and the phase constant $\beta$ can be deduced. Further assume that the object is buried at a depth $d$, and its complex refiectivities are $R_{c} \exp \left\{j \phi_{o c}\right\}$ and $R_{x} \exp \left\{j \phi_{o x}\right\}$ for co-polarized and crosspolarized backscatter, respectively. Under these circumstances, 


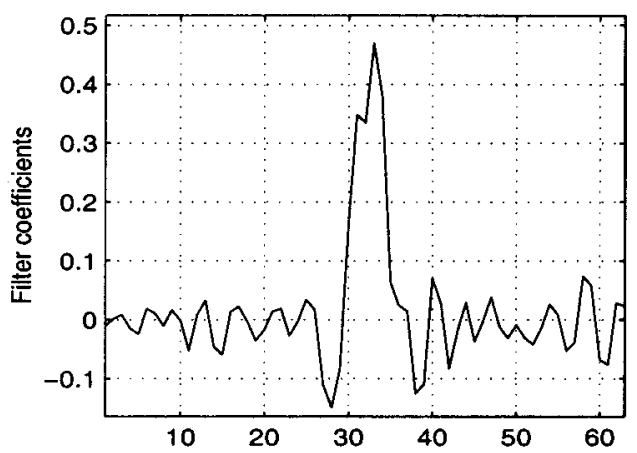

(a)

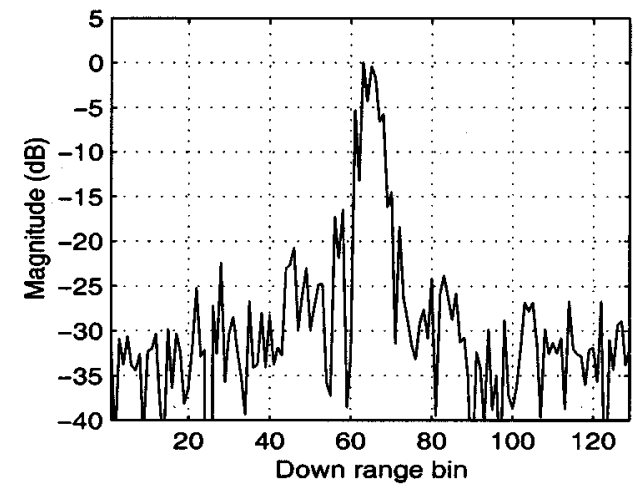

(b)

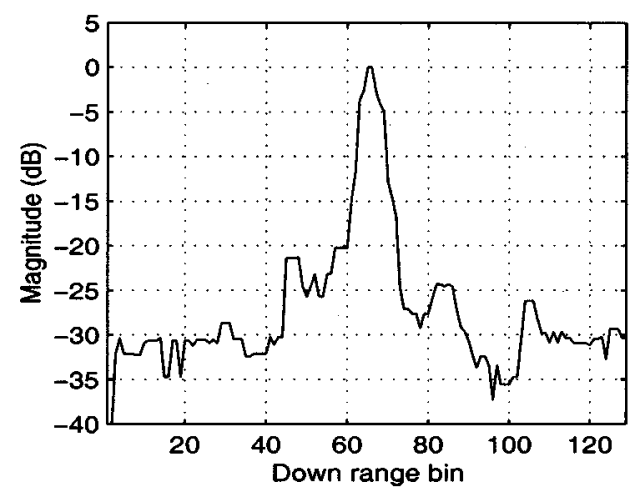

(d)

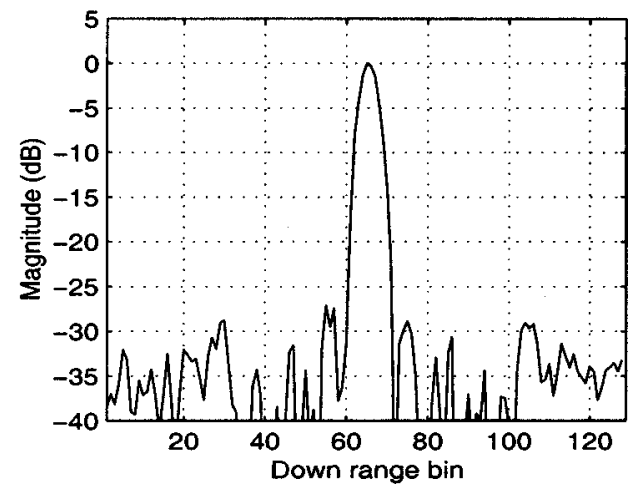

(c)

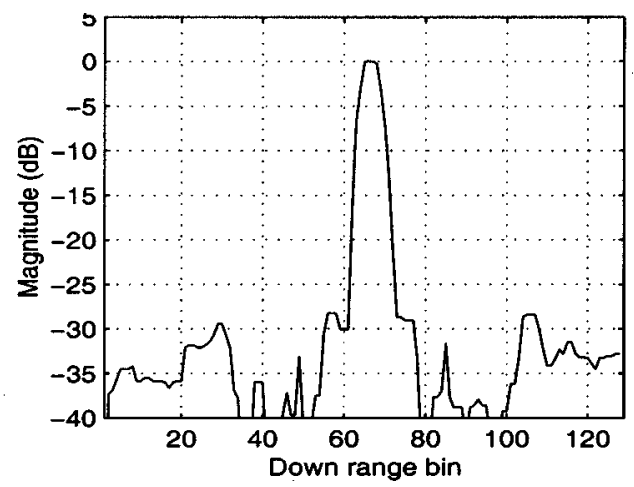

(e)

Fig. 3. True PSFs before and after filtering processing. (a) Derived coefficients for the apodization filter, (b) impulse response before apodization filtering, (c) impulse response after apodization filtering, (d) impulse response after median filtering, and (e) impulse response after apodization and median filtering.

we can show that the outputs of the logarithmic amplifiers AMP 7 and AMP 6 are, respectively

$$
P_{r c}=K R_{c}^{2}
$$

and

$$
P_{r x}=K R_{x}^{2}
$$

where $K$ is some constant. The ratio of $P_{r x}$ to $P_{r c}$ gives us the depolarization ratio of the buried object $D$ as follows

$$
D=\frac{P_{r x}}{P_{r c}}=\frac{R_{x}^{2}}{R_{c}^{2}} .
$$

Also, the outputs of the I/Q detectors IQD1 and IQD2 are, respectively

$$
\bar{\theta}_{c}=\phi_{o c}+\phi_{K}
$$

and

$$
\bar{\theta}_{x}=\phi_{o x}+\phi_{K}
$$

where $\phi_{K}$ is some constant. The difference between $\bar{\theta}_{c}$ and $\bar{\theta}_{x}$ yields the polarimetric phase angle of the object $\theta_{d}$, as follows

$$
\bar{\theta}_{c}-\bar{\theta}_{x}=\phi_{o c}-\phi_{o d}=\theta_{d} .
$$




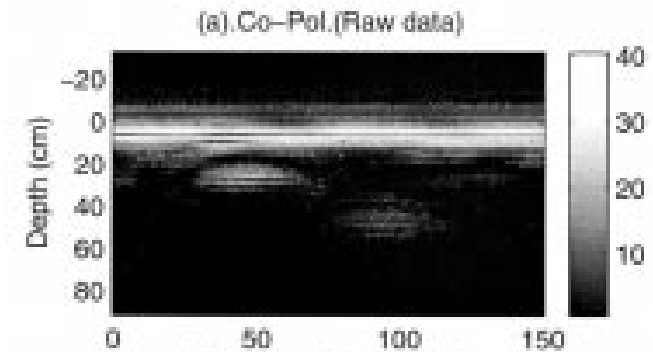

(c).Phase diff_(Raw data)

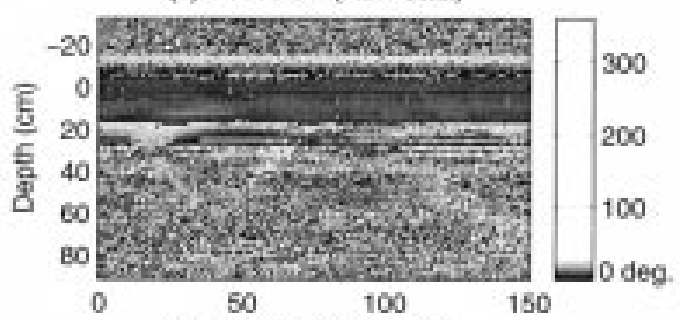

(e).Co-Pol.(Processed)

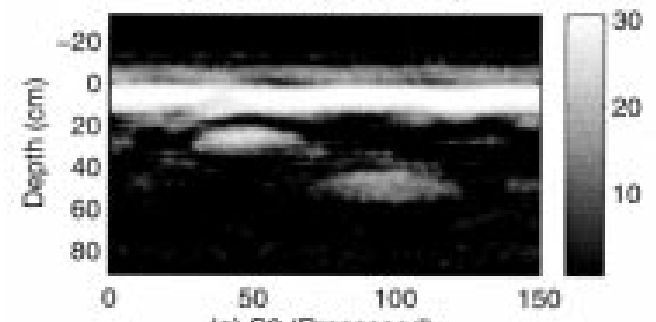

(9).S0 (Processed)

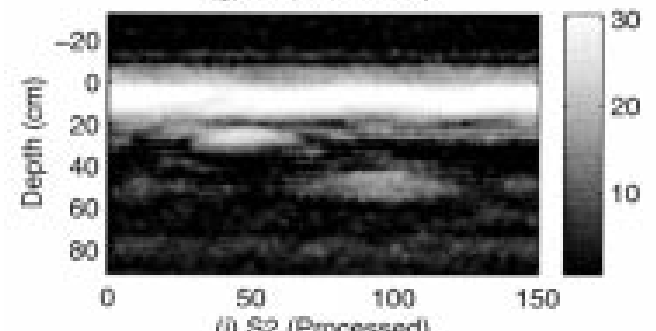

(i).S2 (Processed)

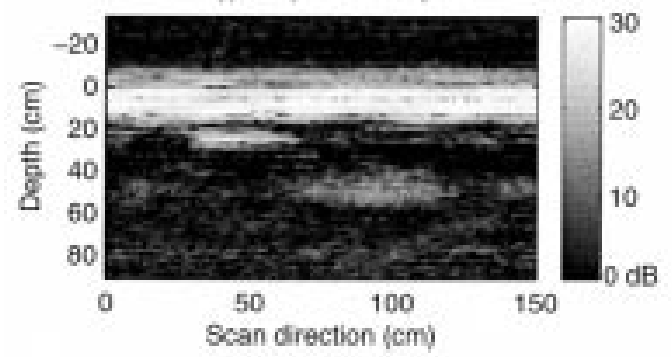

(b).Cross-Pol.(Paw data)

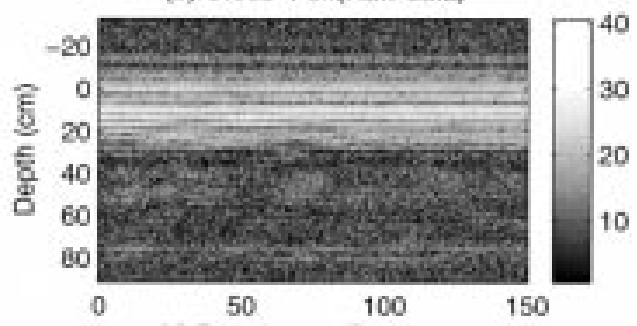

(d).Depol. ratio (Raw data)

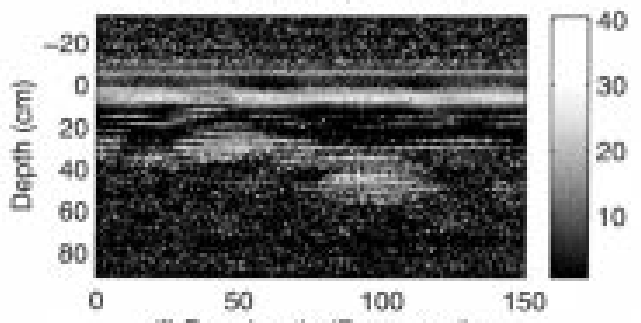

(1).Depol. ratio (Processed)

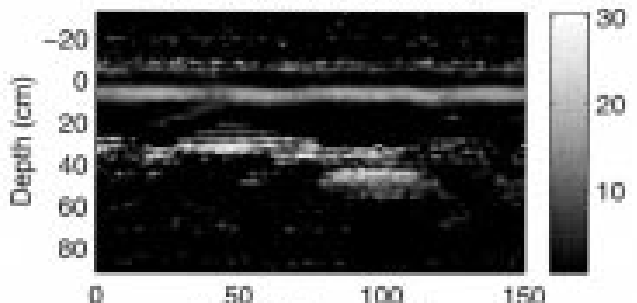

(h).S1 (Processed)
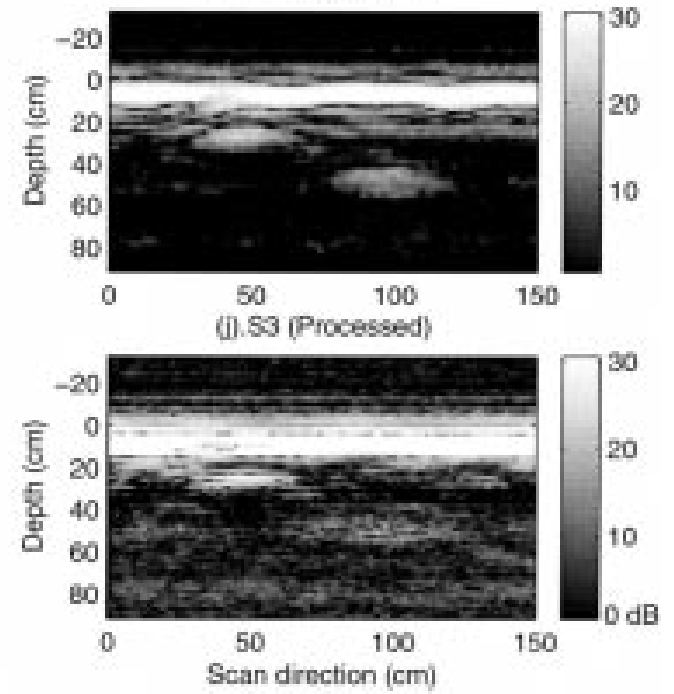

Fig. 4. Raw and processed images: two metallic plates, round and square, with depths 17.8 and $43.2 \mathrm{~cm}$, respectively, horizontal separation $25.4 \mathrm{~cm}$. (a)-(d) Raw images. (a) Co-polarized magnitude, (b) cross-polarized magnitude, (c) polametric phase difference, and (d) depolarization ratio. (e)-(j) Processed images. (e) Co-polarization magnitude, (f) depolarization ratio, (g) $S_{0}$, (h) $S_{1}$, (i) $S_{2}$, and (j) $S_{3}$ images.

The depth resolution $\Delta d$ of the radar system is deduced from its total bandwidth $B$ and the dielectric constant of the soil. This is given by

$$
\Delta d=\frac{c}{2 B \sqrt{\epsilon_{r}^{\prime}}}
$$

For dry soil, $\epsilon_{r}^{\prime} \simeq 3$ and for wet soil $\epsilon_{r}^{\prime} \simeq 25$. Thus, the resolution varies between $8.6 \mathrm{~cm}$ in dry soil and $3 \mathrm{~cm}$ in wet soil.
From the raw data collected by the radar system, we generate images based on the Stokes matrix formulation for facilitating the detection and recognition of targets using the polarimetric information on the buried target [8]. The Stokes vector is a convenient method for representing the polarization state of an electromagnetic wave, and is denoted as [S], given by

$$
[S]=\left[\begin{array}{c}
S_{0} \\
S_{1} \\
S_{2} \\
S_{3}
\end{array}\right] .
$$


(a).Co-Pol,(Aaw data)

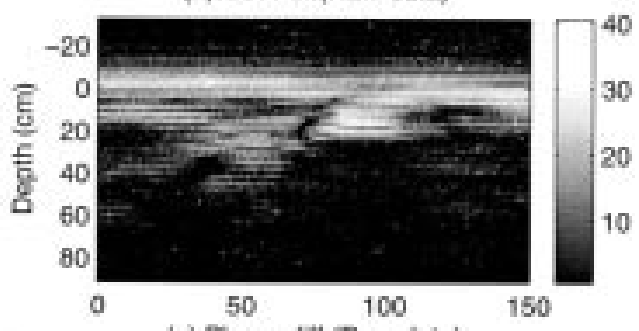

(c).Phase dit. (Raw data)

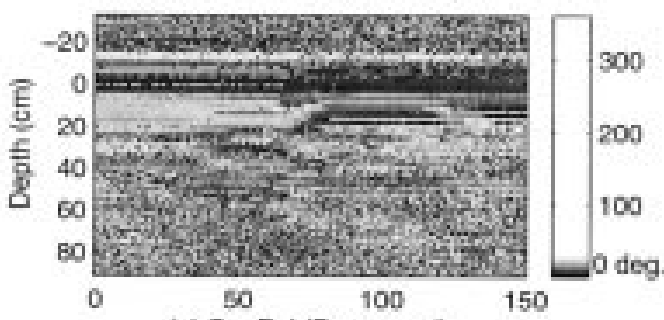

(e).Co-Pol.(Processed)

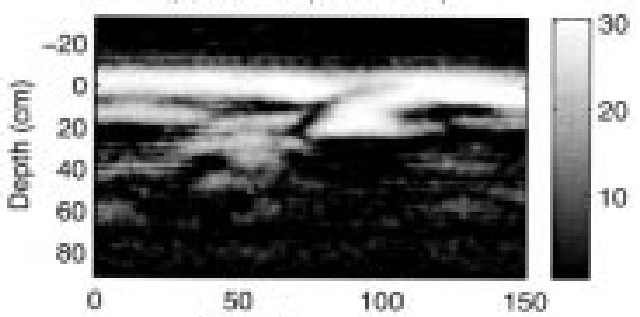

(g).So (Procossed)

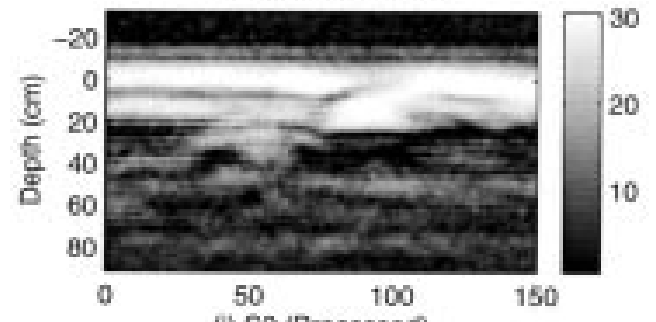

(i). 82 (Processed)

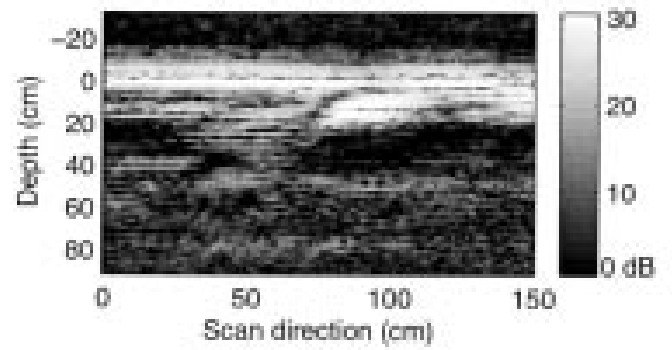

(b).Cross-Pol.(parw data)

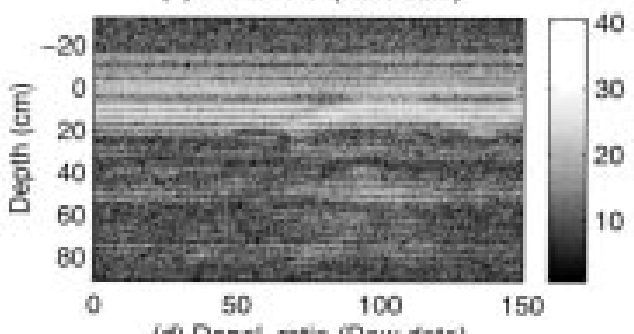

(d).Depol, ratio (Raw data)

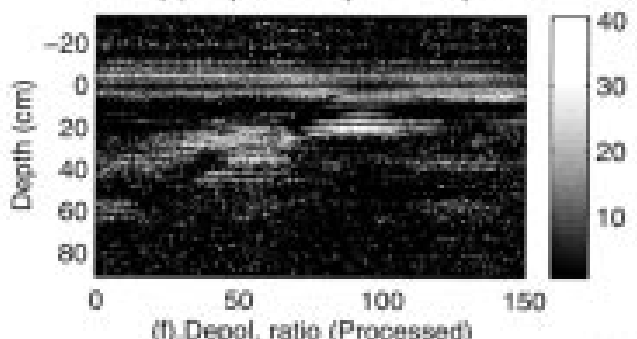

(1). Depol, ratio (Processed)

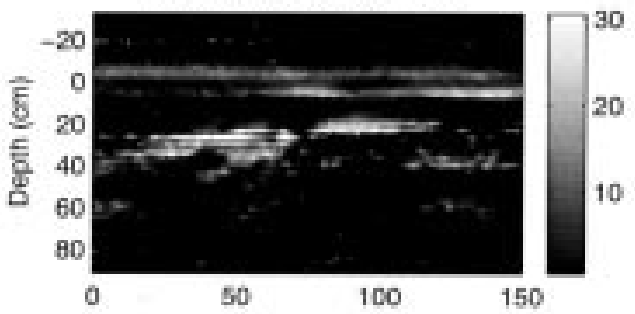

(h) St (Processed)

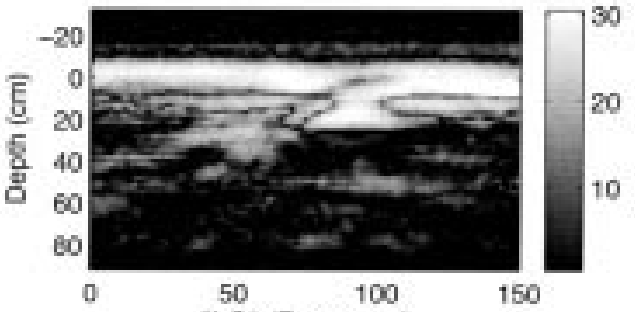

0). .83 (Processed)

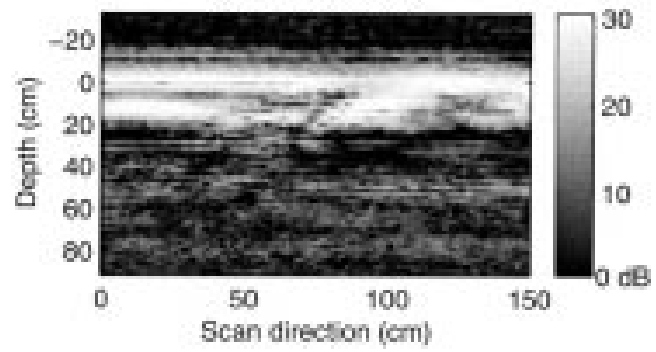

Fig. 5. Raw and processed images. Two round metallic plates, depths 22.9 and $7.6 \mathrm{~cm}$, respectively, horizontal separation $15.2 \mathrm{~cm}$; (a)-(d) Raw images. (a) Co-polarized magnitude, (b) cross-polarized magnitude, (c) polametric phase difference, and (d) depolarization ratio. (e)-(j) Processed images. (e) Co-polarization magnitude, (f) depolarization ratio, (g) $S_{0}$, (h) $S_{1}$, (i) $S_{2}$, and (j) $S_{3}$ images.

The individual elements of the $[S]$ vector are defined as follows:

$$
\begin{aligned}
& S_{0}=\left|E_{\|}\right|^{2}+\left|E_{\perp}\right|^{2} \\
& S_{1}=\left|E_{\|}\right|^{2}-\left|E_{\perp}\right|^{2} \\
& S_{2}=2\left|E_{\|}\right|\left|E_{\perp}\right| \cos \theta_{d} \\
& S_{3}=2\left|E_{\|}\right|\left|E_{\perp}\right| \sin \theta_{d} .
\end{aligned}
$$

In the above equations, $\theta_{d}$ is the polarimetric phase angle, i.e, the difference between the phase angles of the co-polarized and the cross-polarized reflectivities. Also, $\left|E_{\|}\right|$and $\left|E_{\perp}\right|$ are the electric field amplitudes of the co-polarized and cross-polarized received voltages, whose squared values represent the co-polarized reflected power and cross-polarized reflected power, respectively. We recognize $S_{0}$ as the total reflected power (sum of the co-polarized and cross-polarized reflected power). $S_{1}$ is recognized as the difference between the co-polarized and cross-polarized reflected power. $S_{2}$ is proportional to the cosine of the polarimetric phase angle, while $S_{3}$ is proportional to the sine of the polarimetric phase angle $\theta_{d}$. Both $S_{2}$ and $S_{3}$ 


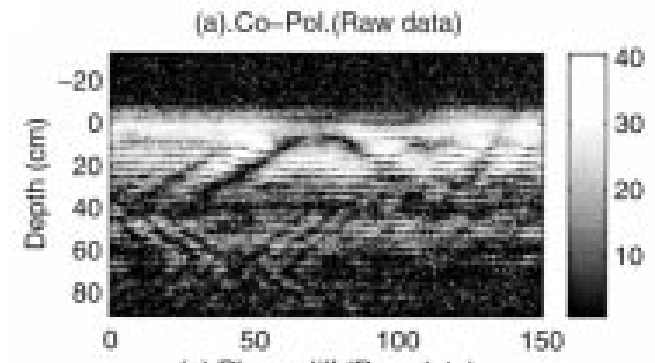

(c) Phase ditt.(Haw data)

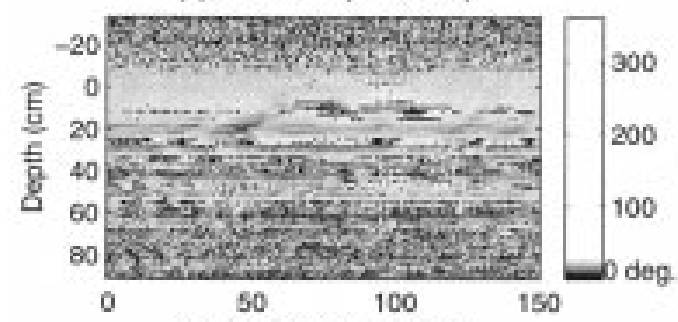

(e).Co-Pol(Processed)
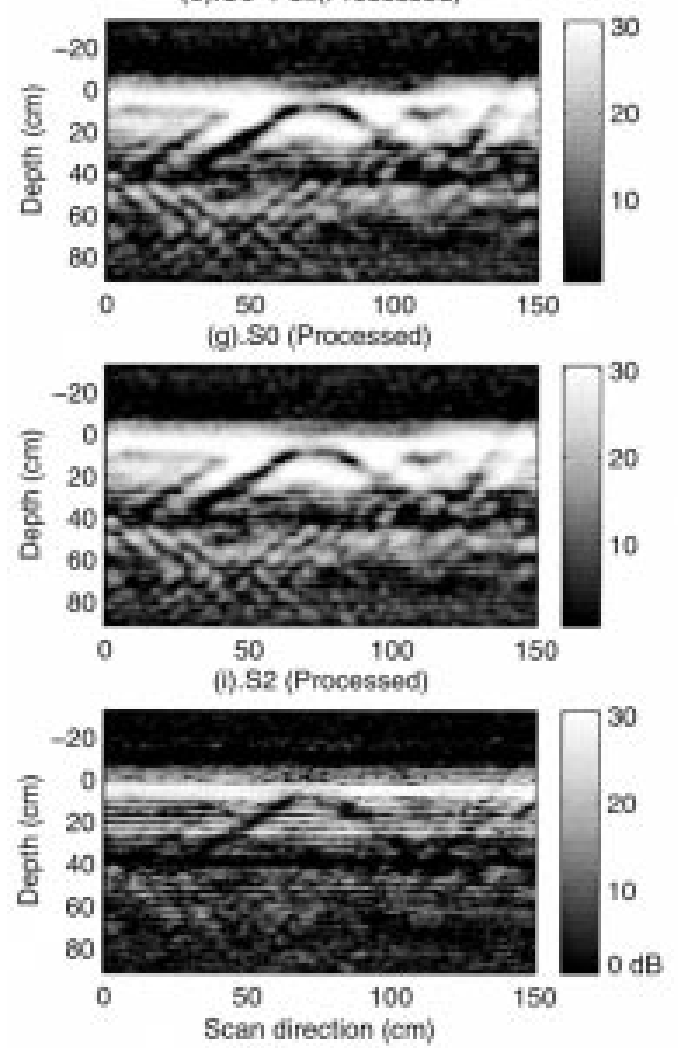

(b).Cross-Pol.(Parw data)

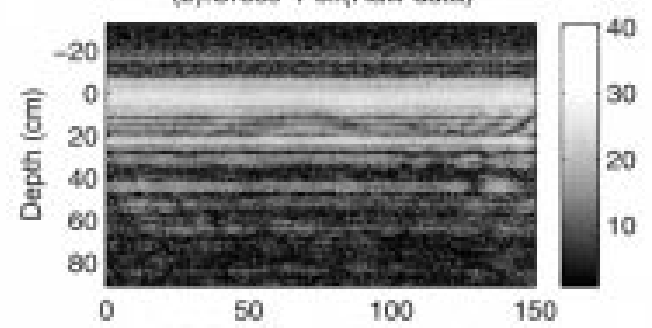

(d) Depol, fatio (fraw data)
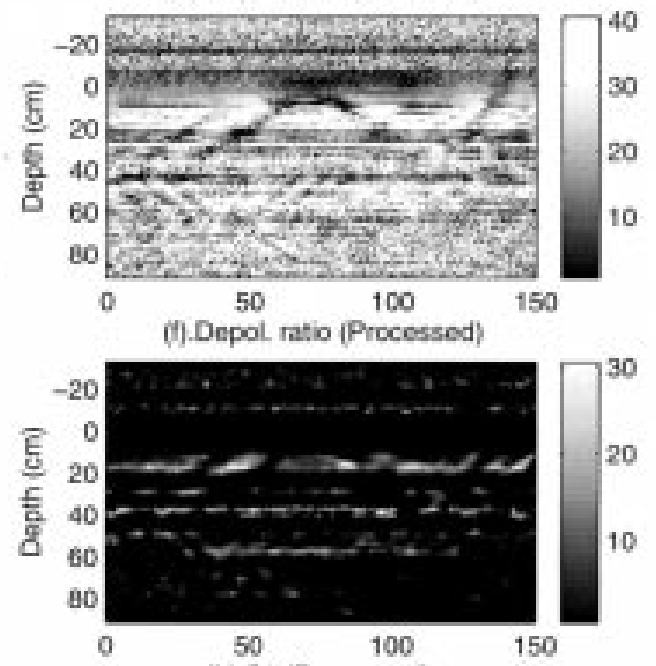

(h) 81 (Processed)
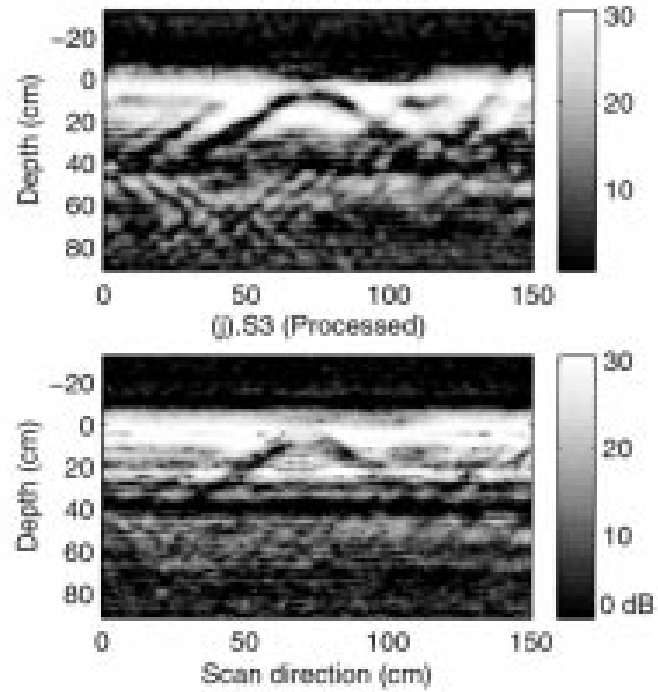

Fig. 6. Raw and processed images: distilled water in 1 gallon plastic container, depth $7.6 \mathrm{~cm}$. (a)-(d) Raw images. (a) Co-polarized magnitude, (b) cross-polarized magnitude, (c) polametric phase difference, and (d) depolarization ratio. (e)-(j) Processed images. (e) Co-polarization magnitude, (f) depolarization ratio, (g) $S_{0}$, (h) $S_{1}$, (i) $S_{2}$, and (j) $S_{3}$ images.

are weighted by the absolute electric field amplitudes of the reflected co-polarized and cross-polarized signals, as can be seen from their definitions. It is also to be noted that

$$
S_{0}^{2}=S_{1}^{2}+S_{2}^{2}+S_{3}^{2} \text {. }
$$

The use of $S_{2}$ and $S_{3}$ greatly enhances the detection of targets, since these parameters move in opposite directions, and thereby provides additional information about the reflected signal. When $S_{2}$ is high, $S_{3}$ is low, and vice versa. Thus, no matter what the polarimetric phase angle is, the target image is bound to show up in either $S_{2}$ or $S_{3}$ or sometimes in both.

\section{Image Processing Results Using Stokes MatriX REPRESENTATION}

In this section, we present the image processing results using typical images culled out of the entire data set acquired by the coherent random noise radar system. Although the whole data set includes data from objects buried in both sand and clay soil, all of the data shown in this paper pertain to objects buried in sand only, since detectability was very poor in clay soil owing to the high loss encountered for signals in the $1-2 \mathrm{GHz}$ fre- 


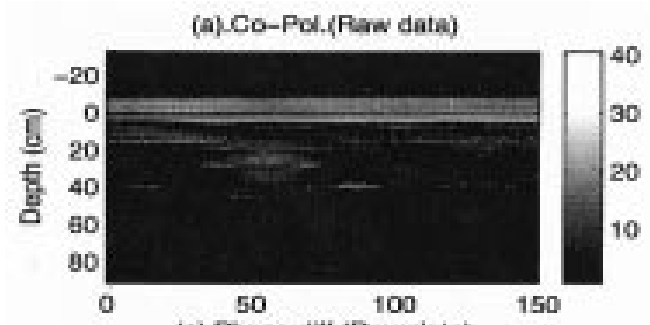

(c) Phase ditt.(Haw data)

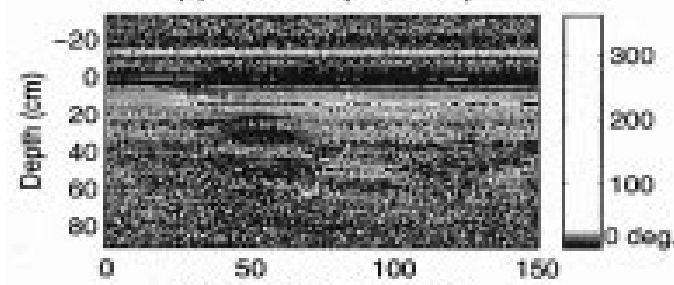

(e).Co-Pol-Processed)

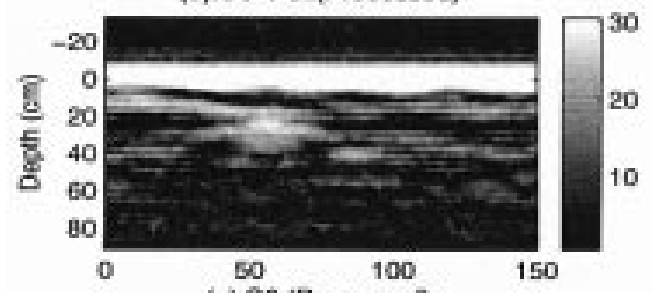

(a).SO (Prooossod)

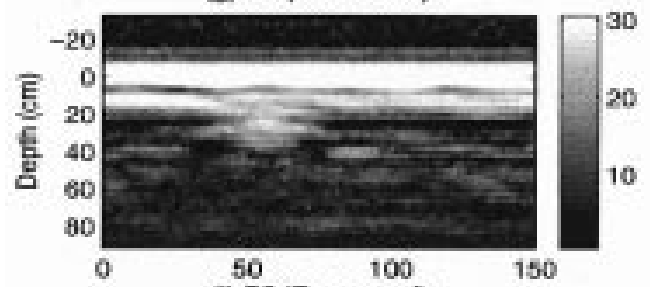

0). 82 (Processed)

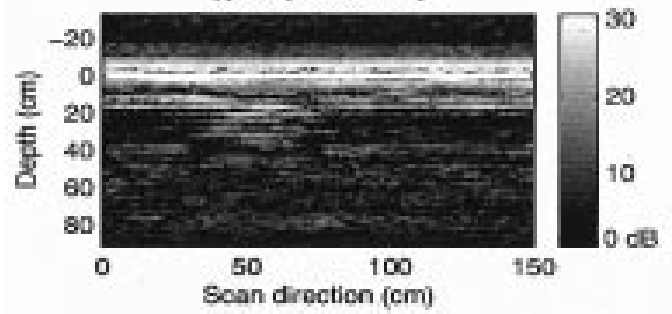

(b).Cross-Pol/(Faw data)

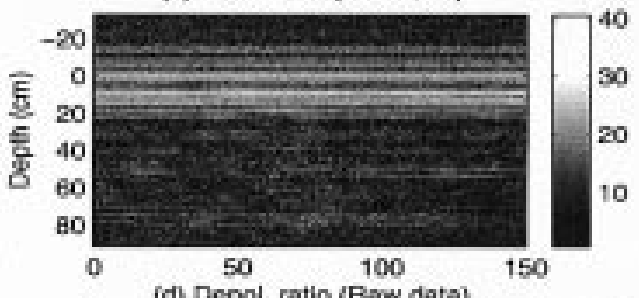

(d) Depol. ratio (Ranw data)

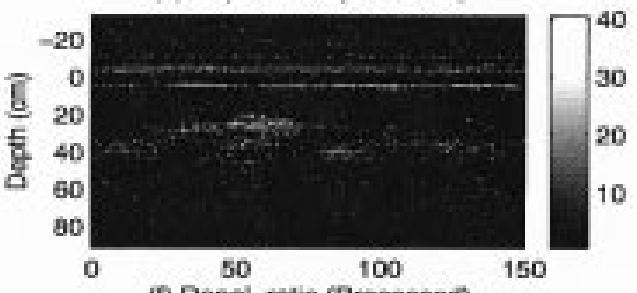

(1).Depol. ratio (Processed)

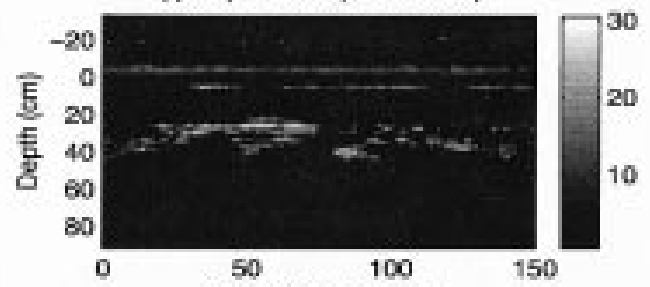

(h) .81 (Procesaed)
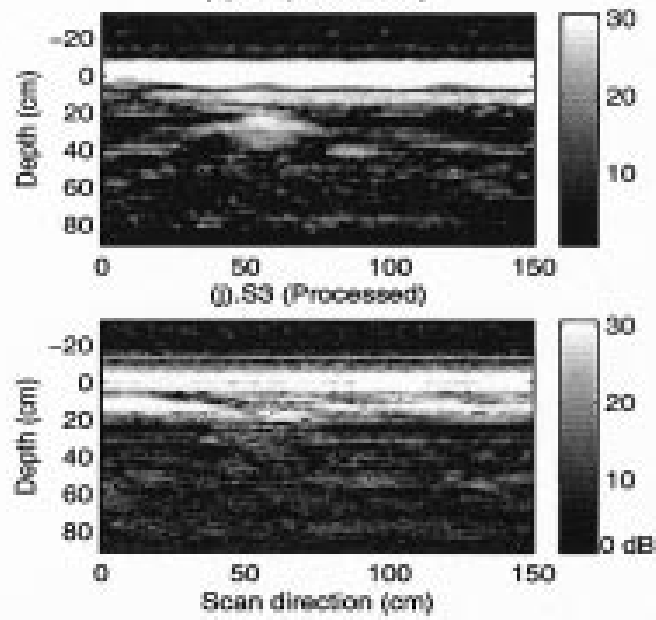

Fig. 7. Raw and processed images: metallic and wooden plates, depths $22.9 \mathrm{~cm}$, horizontal separation $30.5 \mathrm{~cm}$. (a)-(d) Raw images. (a) Co-polarized magnitude, (b) cross-polarized magnitude, (c) polametric phase difference, and (d) depolarization ratio. (e)-(j) Processed images. (e) Co-polarization magnitude, (f) depolarization ratio, (g) $S_{0}$, (h) $S_{1}$, (i) $S_{2}$, and (j) $S_{3}$ images.

quency range. In order to ensure detectability in clay soil, higher transmit power is necessary.

\section{A. Field Test Set-Up}

The system was used to gather data from an assortment of buried objects in a specially designed sand box. The sand box was $3.5 \mathrm{~m}$ long, $1.5 \mathrm{~m}$ wide, and $1 \mathrm{~m}$ deep. The polarimetric random noise radar was operated and controlled by a personal computer (PC), and the data acquired were stored in the hard drive in real time. The radar antennas were scanned over the surface as data were collected continuously. A variety of targets that were buried included metallic as well as nonmetallic objects of different sizes and shapes that mimicked land mines and other objects.
From the raw data, the system produces four images corresponding the co-polarized received amplitude, cross-polarized received amplitude, polarimetric phase difference between the orthogonally polarized received signals, and the depolarization ratio. The raw image data were then used for postprocessing by various image processing algorithms.

\section{B. Image Processing}

The image processing procedure for detection enhancement is as follows. First, the raw data from the radar measurements were preprocessed to suppress the high range sidelobes using an apodization filter [9]. Next, the apodization filtered data were used to calculate the Stokes matrix elements and the depolarization ratio. These data were then used to form the polarimetric 


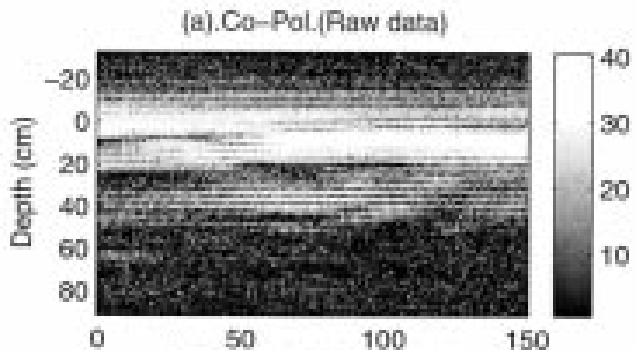

(c).Phase diff.(Raw data)

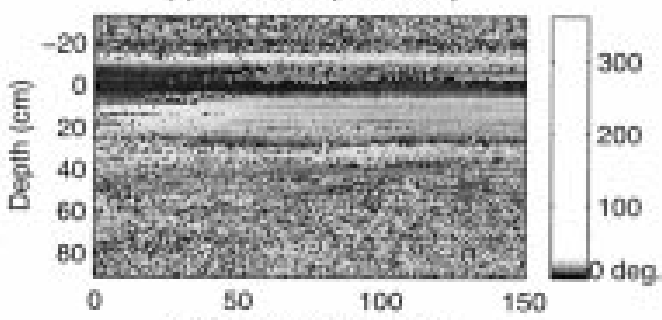

(e).Co-Pol.(Processed)

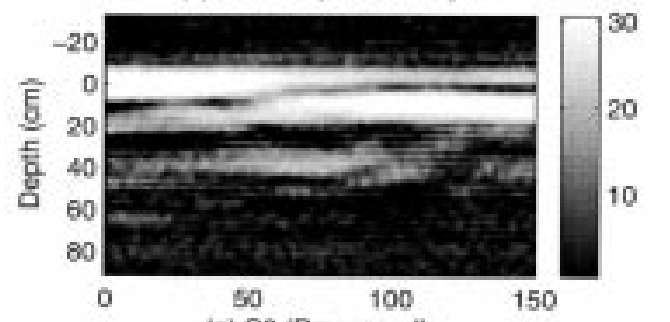

(g) SO (Procossod)

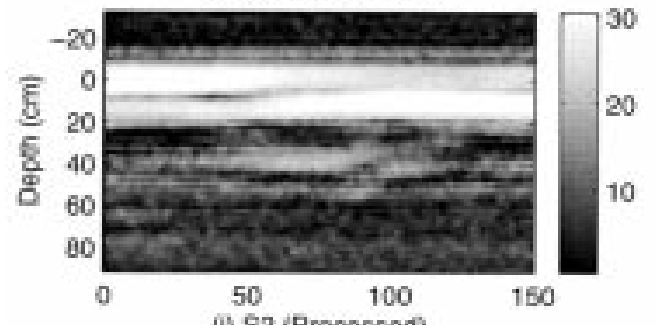

(1). 82 (Processed)

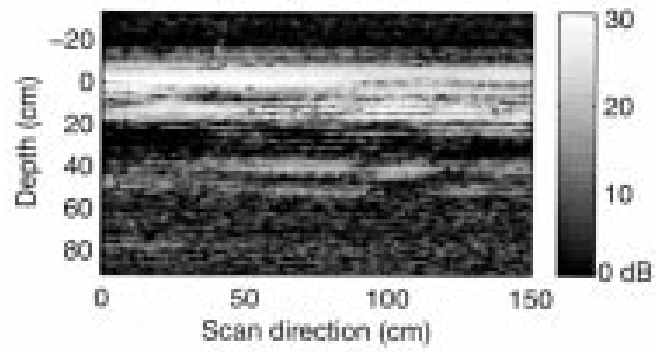

(b).Cross-Pol.(Raw data)

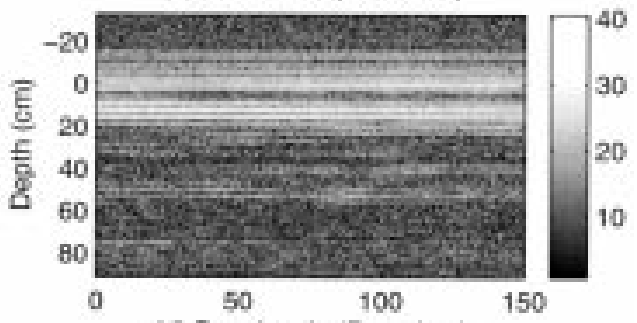

(d).Dopol. ratio (Rare data)

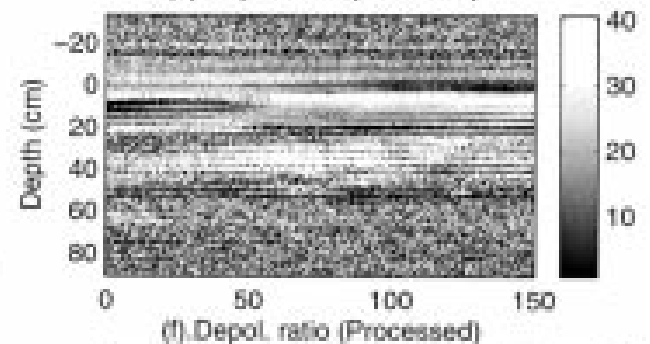

(1). Depol ratio (Processed)

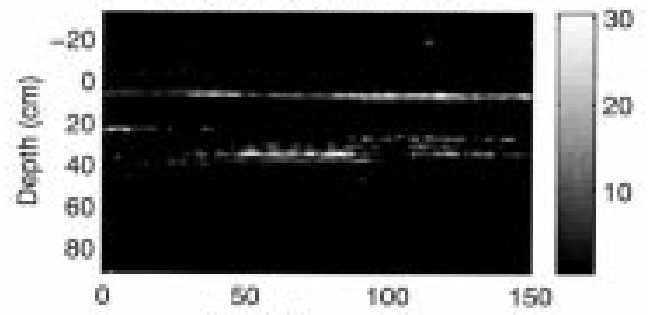

(1)) S1 (Processed)

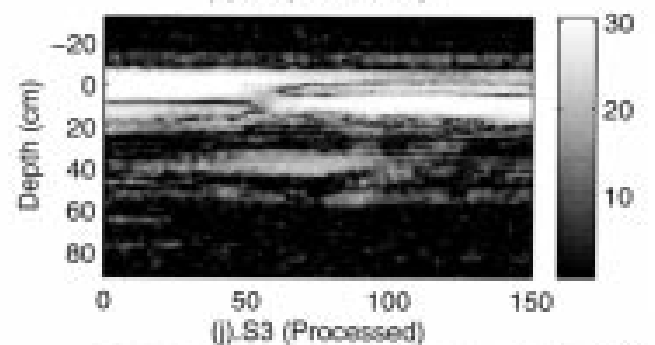

(j) 83 (Processed)

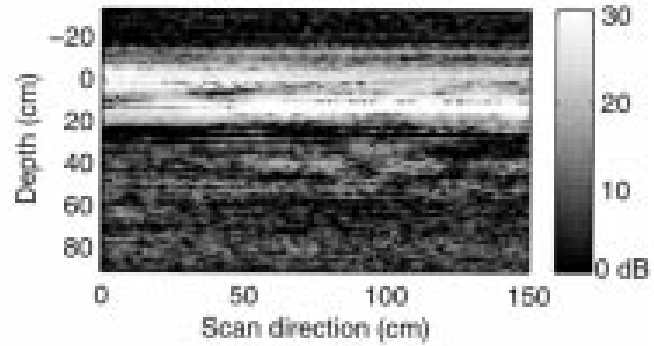

Fig. 8. Raw and processed images: metallic pipe, parallel to both transmit polarization and scan direction, depth $30.5 \mathrm{~cm}$. (a)-(d) Raw images. (a) Co-polarized magnitude, (b) cross-polarized magnitude, (c) polametric phase difference, and (d) depolarization ratio. (e)-(j) Processed images. (e) Co-polarization magnitude, (f) depolarization ratio, (g) $S_{0}$, (h) $S_{1}$, (i) $S_{2}$, and (j) $S_{3}$ images.

Stokes matrix images and depolarization ratio images. Lastly, median filtering and thresholding algorithms were applied to the images to obtain the enhanced Stokes matrix and depolarization ratio images for detection and identification purposes. The concept of apodization filtering for sidelobe suppression is briefly reviewed below.

It was recognized that the impulse response (IR) or the point spread function (PSF) of the radar system is responsible for the high range sidelobes. If we can reduce the sidelobes of the PSF somehow, then the target response or image contaminated by the sidelobes can be restored.
In conventional Fourier harmonic analysis, the frequency domain data is usually multiplied by taper functions [10]. By Fourier transforming the windowed data, the sidelobes resulting from the rectangular window are greatly reduced. In our noise radar system, data sampling is completed in time domain directly. This makes it impossible to reduce the sidelobes by using the same technique as in conventional harmonic analysis. However, by noting that a multiplication in frequency domain is equivalent to a convolution in time domain, a different filtering technique can be developed. This technique is called apodization filtering. By apodization filtering, we mean that, 


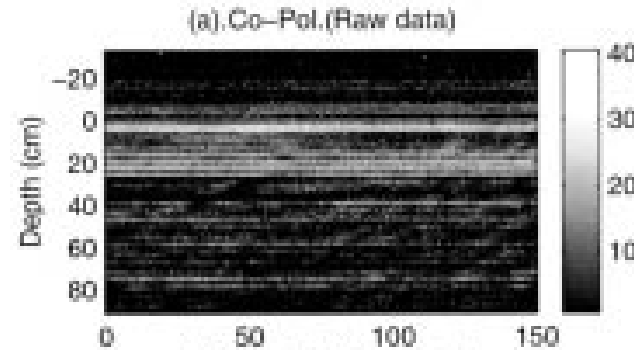

(c).Phase diff. (Raw data)

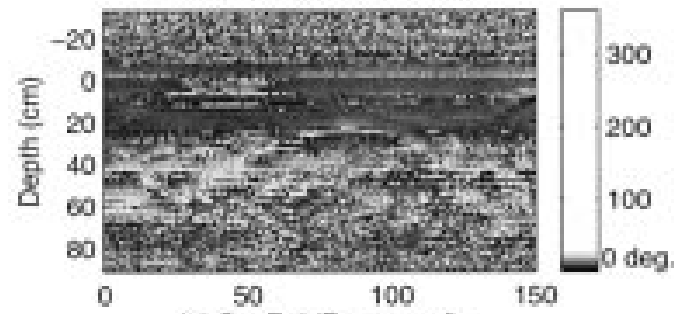

(ब).Co-Pol.(Processed)

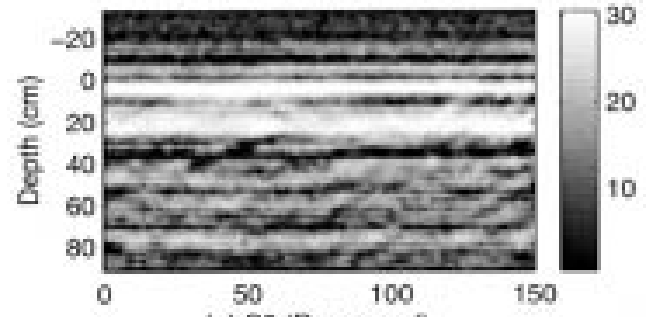

(g).50 (Processed)
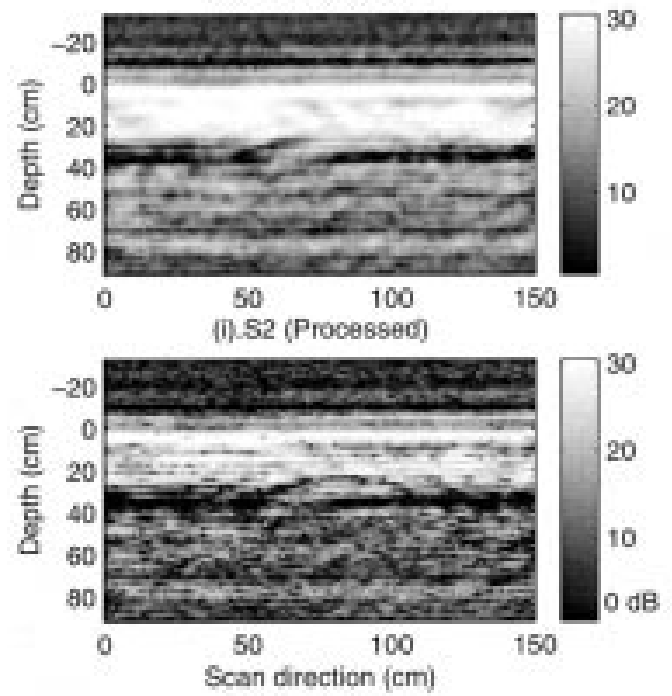

(b).Cross-Pol.(Raw data)

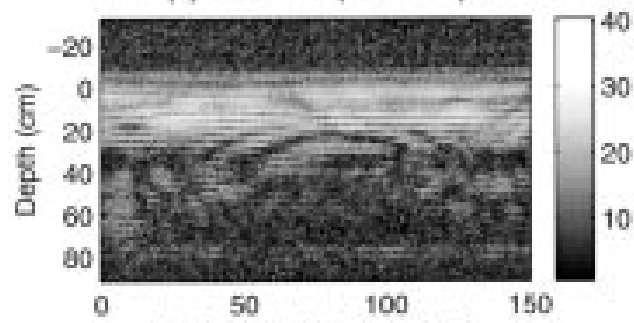

(d).Dopol, ratio (Raw data)

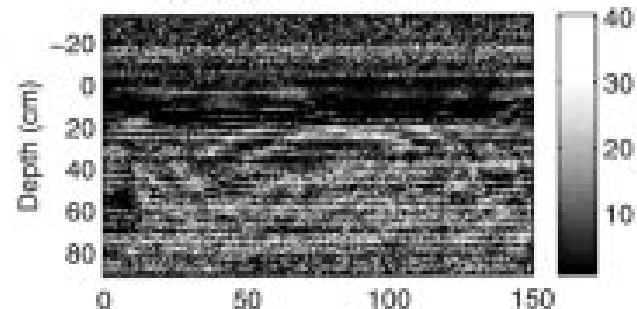

0).Depol. ratio (Processed)

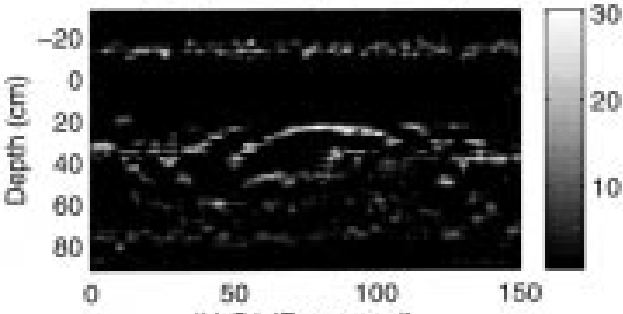

(h).S1 (Processed)
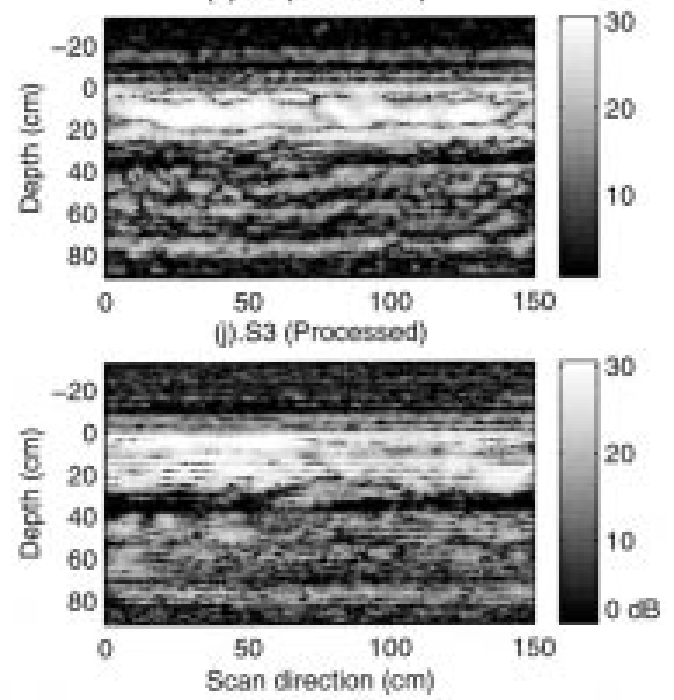

Fig. 9. Raw and processed images: metallic pipe, parallel to transmit polarization, perpendicular to scan direction, depth 30.5 cm. (a)-(d) Raw images. (a) Co-polarized magnitude, (b) cross-polarized magnitude, (c) polametric phase difference, and (d) depolarization ratio. (e)-(j) Processed images. (e) Co-polarization magnitude, (f) depolarization ratio, (g) $S_{0}$, (h) $S_{1}$, (i) $S_{2}$, and (j) $S_{3}$ images.

we are going to find a special filtering function, such that by convolving the time domain data with this filtering function, a new response with much lower sidelobes is obtained. Thus, our objective is to find such a filtering function $T(\tau)$ so that the filtered PSF $P_{T}(\tau)$ given by

$$
P_{T}(\tau)=P(\tau) \otimes T(\tau)
$$

has much lower sidelobes than the original PSF, $P(\tau)$.

To find the filtering function $T(\tau)$, we set $P_{T}(\tau)$ to be the expected PSF. Then the filter coefficient $T(\tau)$ can be derived from the solution of (17). The digitized version of (17) is a set of linear equations. An iterative procedure called the projection method [11] was used to solve the above set of equations.

Fig. 3 illustrates the real impulse response functions of the radar system before and after apodization filtering, where (a) is the derived coefficients for the apodization filter, (b) is the impulse response before apodization filtering, (c) is the impulse response after apodization filtering, (d) is the impulse response after median filtering, and (e) is the impulse response after apodization and median filtering. This example shows that, by using an apodization filter, there is a sidelobe improvement 
of about $10 \mathrm{~dB}$ to the original PSF. This leads to an essential enhancement of the image contrast.

\section{Results}

In the remainder of this section are presented data sets processed using the Stokes matrix formulation. In Figs. 4-9, for each data set, the raw and processed images are displayed in the same way as follows. The raw image data for each measurement are first displayed in the format as shown in (a)-(d). These consist of (a) the co-polarized received magnitude (power), (b) the cross-polarized received magnitude (power), (c) the phase difference of the received co-polarized and cross-polarized signals, and (d) the depolarization ratio. The raw I and Q-channel detector outputs pertaining to the co-polarized and the cross-polarized received signals are used in the computation of the polarimetric phase difference. The apodization filtered co-polarization and depolarization ratio images are then shown in (e) and (f), respectively. Lastly, (g)-(j) show, respectively, the Stokes matrix images $S_{0}, S_{1}, S_{2}$, and $S_{3}$. These are calculated from the apodization filtered amplitude and phase data using (12)-(15) developed in Section III.

1) Detection of Multiple Objects: In Fig. 4, the ability of the radar system to distinguish between objects separated in depth as well as in the lateral direction is demonstrated. Fig. 4 shows the images pertaining to two dissimilar metallic objects, a round metal plate $23 \mathrm{~cm}$ dia $\times 2 \mathrm{~cm}$ thick, and a square metal plate $27 \mathrm{~cm}$ side $\times 1 \mathrm{~cm}$ thick, separated by $25.4 \mathrm{~cm}$ and buried at different depths of $17.8 \mathrm{~cm}$ and $43.2 \mathrm{~cm}$, respectively, i.e., a depth separation of $25.4 \mathrm{~cm}$. The co-polarized reflectivity image in Fig. 4(a) clearly shows the presence of the two objects, but the objects are not observed in the cross-polarized reflectivity image. We also observe that the presence of the object can be clearly discerned in three of the Stokes matrix images, as can be seen in Fig. 4(g)-(i). This indicates that the polarimetric random noise radar does succeed in detecting metallic objects buried at depths as much as about $50 \mathrm{~cm}$ ( 20 inches) in relatively dry sandy soil. In this case, there is no major advantage in polarimetric processing. However, the objects are apparent in the Stokes images.

2) Detection of Near-Surface Objects: Detection of objects on the ground surface or buried very close to the ground surface is a challenging problem because of the high reflectivity due to the air-ground interface. It is expected that substantial blurring would occur so as to make object detection difficult, if not impossible.

Fig. 5 pertains to two object detection, especially when one of the objects is buried very close to the ground surface. In this example, two round metallic plates are buried at depths of 22.9 $\mathrm{cm}$ and $7.6 \mathrm{~cm}$, respectively. It must be noted that the range resolution in dry sandy soil, assuming a soil dielectric constant of about 4 , is approximately $7.5 \mathrm{~cm}$ and therefore, this condition is actually testing the limits of the system resolution. From Fig. 5, we see that the metallic object at 7.6-cm depth is clearly seen in the reflectivity and depolarization, as well as the Stokes images.

3) Detection of Non-Metallic Objects: Fig. 6 shows the image of distilled water (high dielectric constant) contained in a plastic container of approximate size $25 \mathrm{~cm} \times 15 \mathrm{~cm} \times 15 \mathrm{~cm}$. This object was buried at 7.6-cm depth (close to the surface).
The object cannot be clearly recognized in the raw images, but the characteristic hyperbolic feature (similar to the images obtained using impulse radars) is clearly seen in the processed images.

Fig. 7 shows the image of two plates, one metallic and the other wooden, both buried at $22.9 \mathrm{~cm}$ depth, separated by 30.5 $\mathrm{cm}$. From Fig. 7, we observe the metallic object, but not the wooden object in the reflectivity images. However, both objects appear in the depolarization ratio image (low depolarization). This indicates that the process of computing the depolarization assists in enhancing the detectability of nonmetallic objects, since the same amount of signal loss exists in the co-polarized and the cross-polarized reflected signals.

4) Detection of Polarization-Sensitive Objects: In order to study the performance of the system for the detection of long and slender objects, the images of a 6-cm diameter metal pipe of $85-\mathrm{cm}$ length buried at a depth of $30.5 \mathrm{~cm}$ were acquired. These images were obtained for combinations of target orientation parallel and perpendicular to transmit electric field vector and to the scan direction.

Fig. 8 shows that when the pipe orientation is parallel to the transmit polarization and the scan direction, it is clearly discernible in the co-polarized reflectivity and depolarization images. This is due to the long interaction time that occurs during scanning, since the target is oriented along the scan axis. The pipe can also be detected from the Stokes images $S_{0}$ through $S_{2}$. When the pipe is rotated by $90^{\circ}$ to make it perpendicular to the scan direction but keeping it parallel to the transmit polarization, we find that it is still detectable, as shown in Fig. 9. However, it now acts more or less like a point target, since there is minimal interaction time during scanning. We note the characteristic hyperbolic response in the co-polarized reflectivity and depolarization ratio in Fig. 11(b) and (d), as also in the Stokes image $S_{2}$ and $S_{3}$.

On the other hand, when the pipe was buried so that its orientation was orthogonal to the transmit electric field vector, it was observed that the pipe was virtually undetectable.

In summary, a long slender object can be detected, irrespective of its orientation with respect to scan direction, as long as the transmit polarization is parallel to the object orientation. This indicates that a dual-polarized transmitter, i.e., one that simultaneously transmits and receives vertical and horizontal polarized signals, can easily detect such an object.

\section{CONCLUSIONS}

In this paper, we have demonstrated the potential of random noise polarimetry for high-resolution subsurface probing applications. This unique concept synergistically combines the advantages of a random noise ultrawideband waveform with the power of coherent processing to provide a powerful technique for obtaining high resolution images. Use of the apodization filtering technique and Stokes matrix image representation enhances the detection of difficult targets over the conventional images. Other applications being investigated that exploit the coherency in the system include interferometric (using spaced antennas) and synthetic aperture radar (SAR) techniques to sharpen the azimuth resolution. In addition, random noise 
polarimetry can be used in foliage penetration (FOPEN) radar systems by operating at lower frequencies, typically in the 250-500 MHz frequency range.

\section{ACKNOWLEDGMENT}

The authors would like to thank P. Hoffmeyer, S. Hudson, and K. Roesler for technical assistance and support during the fabrication and testing of the radar system, and P. Hirsave during image processing and analysis.

\section{REFERENCES}

[1] D. J. Daniels, D. J. Gunton, and H. F. Scott, "Introduction to subsurface radar," Proc. Inst. Elect. Eng. F, vol. 135, pp. 278-320, Aug. 1988.

[2] T. Suzuki and I. Arai, "Advances in underground radar," IEICE Trans., vol. E74, no. 1, 1991

[3] L. Peters, Jr., J. J. Daniels, and J. D. Young, "Ground penetrating radar as a subsurface environmental sensing tool," Proc. IEEE, vol. 82, pp. 1802-1822, Dec. 1994

[4] T. Moriyama, H. Kashara, Y. Yamaguchi, and H. Yamada, "Advanced polarimetric subsurface FM-CW radar," IEEE Trans. Geosci. Remote Sensing, vol. 36, pp. 725-731, May 1998.

[5] D. A. Noon, I. D. Longstaff, and R. J. Yelf, "Advances in the development of step frequency ground penetrating radar," in Proc. 5th Intl. Conf. Ground Penetrating Radar, ON, Canada, June 1994, pp. 117-132.

[6] I. P. Theron, E. K. Walton, and S. Gunawan, "Compact range radar cross-section measurements using a noise radar," IEEE Trans. Antennas Propagat., vol. 46, pp. 1285-1288, Sept. 1998.

[7] R. M. Narayanan, Y. Xu, P. D. Hoffmeyer, and J. O. Curtis, "Design, performance, and applications of a coherent ultrawideband random noise radar," Opt. Eng., vol. 37, pp. 1855-1869, June 1998.

[8] T. Moriyama, M. Nakamura, Y. Yamaguchi, H. Yamada, and W. M. Boerner, "Classification of targets buried in the ground by radar polarimetry," IEICE Trans. Commun., vol. E82B, pp. 951-957, June 1999.

[9] M. Muller and G. J. Brakenhoff, "Apodization and the point-spread autocorrelation function," Appl. Opt., vol. 36, pp. 2755-2762, May 1997.

[10] F. J. Harris, "On the use of windowd for harmonic analysis with the discrete Fourier transform," Proc. IEEE, vol. 66, pp. 51-83, Jan. 1978.

[11] T. S. Huang, D. A. Barker, and S. P. Berger, "Iterative image restoration," Appl. Opt., vol. 14, pp. 1165-1168, May 1975.

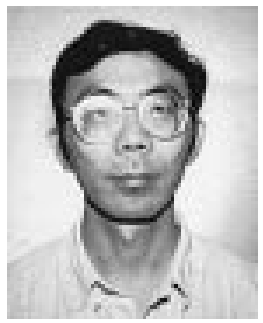

Yi Xu received the B.S.E.E. degree from Beijing Normal University, Beijing, China, in 1982, the M.S.E.E. degree from Hangzhou University, Hangzhou, China, in 1989, and the Ph.D. degree from the University of Nebraska, Lincoln, in 1997, all in electrical engineering.

From 1993 to 1997, he was a Research Assistant with the Environmental Remote Sensing Laboratory (ERSL), University of Nebraska. As part of his doctoral dissertation, he developed and tested an ultrawideband random noise radar system operating in the $1-2 \mathrm{GHz}$ frequency range as a ground penetration radar (GPR) system. From 1997 to 2000, he worked as a programmer at SITEL corp. Currently, he is a software engineer with Agilent Technologies, Santa Rosa, CA, developing object-oriented programming for the radio frequency integrated circuit (RFIC) test system.

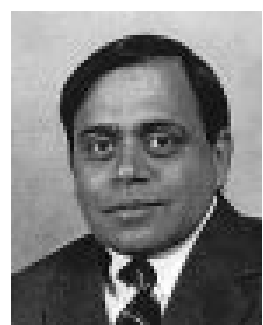

Ram M. Narayanan (S'83-M'88-SM'91-F'01) received the $\mathrm{B}$.Tech. degree in electrical engineering from the Indian Institute of Technology, Madras, India, in 1976, and the Ph.D. degree in electrical and computer engineering from the University of Massachusetts, Amherst, in 1988.

He is currently a Professor of electrical engineeringwith the University of Nebraska, Lincoln. He has been working in the area of radar and laser remote sensing for over 15 years, and is primarily interested in instrument and algorithm development. Among his accomplishments have been the development of high resolution imaging using random noise radar, the development of mid-infrared laser remote sensing technology, and the characterization of information content in remotely sensed images. He is currently establishing an Airborne Remote Sensing Facility at the University of Nebraska with funding from the National Science Foundation. He has published over 30 papers in refereed journals, and presented over 100 conference papers.

Dr. Narayanan is active in the IEEE Geoscience and Remote Sensing Society.

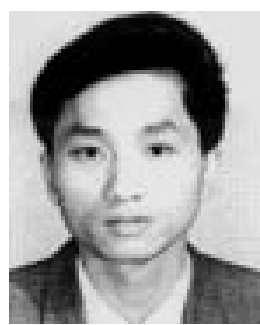

Xiaojian Xu received the B.S. degree in electrical engineering from Hefei University of Technology, Hefei, China, and the M.S. degree in electrical engineering from Beijing Institute of Environmental Features (BIEF), Beijing, China, in 1983 and 1986, respectively.

He joined BIEF in 1986, where his major research work covered electromagnetic and microwave technologies, especially in the field of microwave imaging, electromagnetic modeling and measurements, and image processing. From May to November 1994, he was a Visiting Scholar, Imperial College of Science, Technology and Medicine, London, U.K. Since June 1999, he has been with the Environmental Remote Sensing Laboratory, Department of Electrical Enginnering, University of Nebraska, Lincoln. His major research interests are radar imaging, remote sensing image processing, and electromagnetic scattering and propagation modeling. His recent activities have included developing the UNL coherent ultra-wideband random noise radar and utilizing this system for foliage and ground penetration studies.

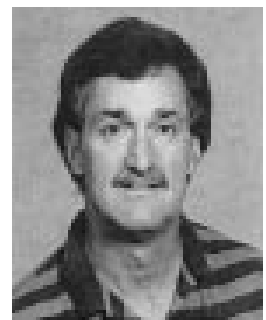

John O. Curtis received the B.S. degree in mechanical engineering from Northeastern University, Boston, MA, in 1971, the M.S. degree in engineering mechancis from Colorado State University, Fort Collins, in 1973, and the Ph.D. in physics from Dartmouth College, Hanover, NH, in 1992.

He has worked as a researcher at the U.S. Army Engineer Waterways Experiment Station, Vicksburg, MS, since 1974. His work assignments have included dynamic finite element simulations of soil-structure interaction problems, thermal camouflage of fixed facilities, and applications of millimeter wave and microwave systems in military environments. Recent interests have focused on laboratory measurements of the complex dielectric properties of soils to support terrain subsurface investigations using geophysical sensors. 